\title{
Acute Right Ventricular Myocardial Infarction by ECG Finding
}

National Cancer Institute

\section{Source}

National Cancer Institute. Acute Right Ventricular Myocardial Infarction by ECG Finding. NCI Thesaurus. Code C102594.

An electrocardiographic finding in leads V1 or V2 of an initial R wave duration greater than or equal to $40 \mathrm{~ms}$, R wave greater than S wave, upright T wave, with accompanying ST elevation, which is suggestive of acute myocardial infarction of the ventricular wall of the left ventricle. Evidence of inferior or lateral myocardial infarction is usually also present. (CDISC) 